\title{
Erratum to: SEOM guidelines for the treatment of gastric cancer 2015
}

\author{
M. Martin-Richard ${ }^{1}$ A. Custodio ${ }^{2}$ - C. García-Girón ${ }^{3}$ C. Grávalos ${ }^{4}$. \\ C. Gomez ${ }^{4}$ P. Jimenez-Fonseca ${ }^{5}$ J. L. Manzano ${ }^{6}$ - C. Pericay ${ }^{7}$ F. Rivera ${ }^{8}$. \\ A. Carrato ${ }^{9}$
}

Published online: 11 February 2016

(C) Federación de Sociedades Españolas de Oncología (FESEO) 2016

\section{Erratum to: Clin Transl Oncol (2015) 17:996-1004 DOI 10.1007/s12094-015-1456-y}

The affiliation of the author A. Custodio was rendered wrongly. The correct affiliation is: Medical Oncology Department, Hospital Universitario La Paz, Madrid, Spain. The authors regret this error and apologize for any inconvenience caused.

The online version of the original article can be found under doi:10.1007/s12094-015-1456-y.

M. Martin-Richard

mmartinri@santpau.cat

1 Medical Oncology Department, Hospital de la Santa Creu I, Sant Pau, 167, 08025 Barcelona, Spain

2 Medical Oncology Department, Hospital Universitario La Paz, Madrid, Spain

3 Medical Oncology Department, Hospital Universitario de Burgos, Burgos, Spain

4 Medical Oncology Department, Hospital Universitario 12 de Octubre, Madrid, Spain

5 Medical Oncology Department, Hospital Universitario Central de Asturias (HUCA), Oviedo, Spain

6 Medical Oncology Department, Hospital Universitari Germans Trias I Pujol de Badalona, Barcelona, Spain

7 Medical Oncology Department, Hospital de SabadellConsorcio Sanitario Parc Taulì, Barcelona, Spain

8 Medical Oncology Department, Hospital Universitario Marqués de Valdecilla, Santander, Spain

9 Medical Oncology Department, Hospital Universitario Ramón y Cajal, Madrid, Spain 